\title{
Profiling metabolic changes in breast cancer with targeted proteomics
}

\author{
Stephan Bernhardt ${ }^{*}$, Devina Mitra ${ }^{1}$, Zita Soons ${ }^{2}$, Rainer König ${ }^{3}$, Martina Vetter ${ }^{4}$, Christoph Thomssen ${ }^{4}$, \\ Eva Kantelhardt ${ }^{4}$, Stefan Wiemann ${ }^{1}$, Ulrike Korf ${ }^{1}$ \\ From Metabolism, Diet and Disease 2014: Cancer and metabolism \\ Washington DC, USA. 28-30 May 2014
}

\section{Background}

Breast cancer is one of the most common causes of death from cancer among women. Significant but yet insufficient progress has been achieved due to the introduction of targeted therapeutics [1]. Nevertheless, advancing personalized medicine requires a detailed and comprehensive molecular characterization, particularly at the protein level. This project aims to advance the understanding of crosstalk between cancer-relevant signaling and metabolism.

\section{Materials and methods}

Based on a constructed flux distribution model, targets where chosen to be analyzed in breast cancer cell lines and tumor samples. The RPPA (reverse phase protein microarray) technology a micro-scaled dot-blot approach is used. RPPA allows to quantify protein expression and activation states in a large panel of samples. In addition, RPPA has a remarkable high sensitivity and is able to analyze many proteins in a single experiment. Therefore this method presents a powerful approach for targeted hypothesis-based proteome research [2].

\section{Results}

As a first step, based on publically available transcript data a model describing the deregulation especially of glutamine and nitrogen metabolism in breast cancer was developed [3]. Key features of our model are currently evaluated by RPPA in cell lines representing the different breast cancer subtypes. Metabolic enzymes confirmed on the cell line level as de-regulated will be evaluated in tumor samples and tested as putative drug targets in in vitro models.

\section{Conclusion}

Based on the bioinformatic model and proteomic validation our aim is to introduce options for new co-treatment concepts that target key metabolic enzymes to harm tumor cells and disable their malignant anabolic processes, as well as energy consumption. The resulting data will provide a basis to calculate new models explaining the metabolic shift in breast cancer cells and to identify signaling molecules that contribute to malignant transformation.

\section{Authors' details}

'Division Molecular Genome Analysis, German Cancer Research Center (DKFZ), 69120, Heidelberg, Germany. 'Maastricht University, 6200, Maastricht, The Netherlands. ${ }^{3}$ Leibniz Institute for Natural Product Research and Infection Biology - Hans Knöll Institute, 07745, Jena, Germany. ${ }^{4}$ University Hospital Halle, 06120, Halle(Saale), Germany.

\section{Published: 28 May 2014}

\section{References}

1. Lin SX, Chen J, Mazumdar M, Poirier D, Wang C, Azzi A, Zhou M: Molecular therapy of breast cancer: progress and future directions. Nat Rev Endocrinol 2010, 6:485-493.

2. Paweletz CP, Charboneau L, Bichsel VE, Simone NL, Chen T, Gillespie JW, Emmert-Buck MR, Roth MJ, Petricoin IE, Liotta LA: Reverse phase protein microarrays which capture disease progression show activation of prosurvival pathways at the cancer invasion front. Oncogene 2001, 20:1981-1989.

3. Curtis C, Shah SP, Chin SF, Turashvili G, Rueda OM, Dunning MJ, Speed D, Lynch AG, Samarajiwa $S, Y$ Yan $Y$, et al: The genomic and transcriptomic architecture of 2,000 breast tumours reveals novel subgroups. Nature 2012, 486:346-352.

\section{doi:10.1186/2049-3002-2-S1-P7}

Cite this article as: Bernhardt et al:: Profiling metabolic changes in breast cancer with targeted proteomics. Cancer \& Metabolism 2014 2(Suppl 1):P7

'Division Molecular Genome Analysis, German Cancer Research Center

(DKFZ), 69120, Heidelberg, Germany

Full list of author information is available at the end of the article 